\title{
The instability of nonseparable complete Erdős spaces and representations in $\mathbb{R}$-trees
}

by

\author{
Jan J. Dijkstra and Kirsten I. S. Valkenburg (Amsterdam)
}

\begin{abstract}
One way to generalize complete Erdős space $\mathfrak{E}_{\mathrm{c}}$ is to consider uncountable products of zero-dimensional $G_{\delta}$-subsets of the real line, intersected with an appropriate Banach space. The resulting (nonseparable) complete Erdős spaces can be fully classified by only two cardinal invariants, as done in an earlier paper of the authors together with J. van Mill. As we think this is the correct way to generalize the concept of complete Erdős space to a nonseparable setting, natural questions arise about analogies between the behaviour of complete Erdös space and its generalizations. The discovery that $\mathfrak{E}_{\mathrm{c}}$ is unstable, by which we mean that the space is not homeomorphic to its infinite power, by Dijkstra, van Mill, and Steprāns, led to the solution of a series of problems in the literature. In the present paper we prove by a different method that our nonseparable complete Erdös spaces are also unstable. Another application of $\mathfrak{E}_{\mathrm{c}}$ is that it is homeomorphic to the endpoint set of the universal separable $\mathbb{R}$-tree. Our standard models can also be represented as endpoint sets of more general $\mathbb{R}$-trees, but some universality properties are lost.
\end{abstract}

1. Introduction. Let $\lambda, \kappa$ be arbitrary infinite cardinal numbers, and let $p \geq 1$. Recall the (possibly nonseparable) Banach space $\ell_{\kappa}^{p}$, given by

$$
\ell_{\kappa}^{p}=\left\{x=\left(x_{\alpha}\right)_{\alpha \in \kappa} \in \mathbb{R}^{\kappa}: \sum_{\alpha \in \kappa}\left|x_{\alpha}\right|^{p}<\infty\right\},
$$

equipped with the topology generated by the norm $\|x\|=\left(\sum_{\alpha \in \kappa}\left|x_{\alpha}\right|^{p}\right)^{1 / p}$.

For $\omega \leq \lambda \leq \kappa$, let

$$
F_{\alpha}= \begin{cases}\{0\} \cup\{1 / n: n \in \mathbb{N}\} & \text { if } \alpha \in \lambda, \\ \{0,1\}, & \text { if } \alpha \in \kappa \backslash \lambda,\end{cases}
$$

and define the nonseparable complete Erdös space by

$$
\mathfrak{E}_{\mathrm{c}}(\lambda, \kappa)=\left\{x \in \ell_{\kappa}^{1}: \forall \alpha \in \kappa, x_{\alpha} \in F_{\alpha}\right\} .
$$

2010 Mathematics Subject Classification: 54F50, 46B26.

Key words and phrases: complete Erdős space, $\mathbb{R}$-tree, almost zero-dimensional, nonseparable Banach space. 
For $\lambda=\kappa=\omega$, this represents complete Erdös space, $\mathfrak{E}_{\mathrm{c}}$, introduced by Paul Erdős in 1940 [10]. He also introduced Erdös space,

$$
\mathfrak{E}=\left\{x \in \ell_{\omega}^{2}: \forall n \in \omega, x_{n} \in \mathbb{Q}\right\} .
$$

and proved that both $\mathfrak{E}$ and $\mathfrak{E}_{\mathrm{c}}$ are one-dimensional, yet totally disconnected and homeomorphic to their own squares. The spaces $\mathfrak{E}, \mathfrak{E}_{\mathrm{c}}$ and $\mathfrak{E}_{\mathrm{c}}^{\omega}$ were characterized by Dijkstra and van Mill [2, 5, 4] and Dijkstra [1. Nonseparable complete Erdös spaces have analogous properties, as shown by Dijkstra, van Mill, and Valkenburg [7]. That paper concerns also more general spaces defined by

$$
\mathcal{E}_{\mu}=\left\{x \in \ell_{\mu}^{p}: \forall \alpha \in \mu, x_{\alpha} \in E_{\alpha}\right\},
$$

where $\mu$ is another arbitrary infinite cardinal number and the $E_{\alpha}$ are arbitrary zero-dimensional subsets of $\mathbb{R}$. Let $w(X)$ denote the weight of a space $X$ and $l w(X)$ the local weight, which is defined as $\min \{w(U): U \subset X$ nonempty and open $\}$. The values of these cardinal invariants can be easily determined for the space $\mathcal{E}_{\mu}$ whenever the sets $E_{\alpha}$ are given (see [7, Proposition 12]). The following two theorems are the main results in [7]; the symbol $X_{\mathrm{D}}$ denotes the set $X$ equipped with the discrete topology.

Theorem 1. The space $\mathcal{E}_{\mu}$ is homeomorphic to $\mathfrak{E}_{\mathrm{c}}\left(l w\left(\mathcal{E}_{\mu}\right), w\left(\mathcal{E}_{\mu}\right)\right)$ if and only if every $E_{\alpha}$ is a $G_{\delta}$-subset of $\mathbb{R}$ and ind $\mathcal{E}_{\mu}>0$.

TheOREM 2. The space $\mathfrak{E}_{\mathrm{c}}(\lambda, \kappa)$ is homeomorphic to $\mathfrak{E}_{\mathrm{c}} \times\left(\lambda_{\mathrm{D}}\right)^{\omega} \times \kappa_{\mathrm{D}}$.

We claim that our definition of nonseparable complete Erdős spaces forms the right concept that generalizes complete Erdős space to a (possibly) nonseparable setting. The aim of this paper is to further justify this, by considering to what extent some applications of $\mathfrak{E}_{\mathrm{c}}$ can be generalized to $\mathfrak{E}_{\mathrm{c}}(\lambda, \kappa)$. The first application is that $\mathfrak{E}_{\mathrm{c}}$ is unstable, that is, $\mathfrak{E}_{\mathrm{c}} \not \approx \mathfrak{E}_{\mathrm{c}}^{\omega}$ (see Dijkstra, van Mill, and Steprāns [6] and Dijkstra [1]). This result produced solutions to a series of problems in the literature (see [6, 3]). We prove in $\S 3$ that $\mathfrak{E}_{\mathrm{c}}(\lambda, \kappa)$ is not homeomorphic to $\left(\mathfrak{E}_{\mathrm{c}}(\lambda, \kappa)\right)^{\omega}$. Another application is that $\mathfrak{E}_{\mathrm{c}}$ is homeomorphic to the endpoint set of the unique universal separable $\mathbb{R}$-tree, as shown first by Kawamura, Oversteegen, and Tymchatyn [11]. In $\S 4$ we find explicit constructions of $\mathbb{R}$-trees with endpoint set homeomorphic to $\mathfrak{E}_{\mathrm{c}}(\kappa, \kappa)$ and consider some universality properties derived from these constructions.

\section{Preliminaries}

Definition 3. A Hausdorff space (or a topology) is called zero-dimensional if the small inductive dimension is at most 0 , that is, if there is a basis consisting of clopen sets. A Hausdorff space $X$ is called almost zerodimensional if there exists a second topology $\mathcal{W}$ on $X$ that witnesses the 
almost zero-dimensionality of $X$, which means that $\mathcal{W}$ is zero-dimensional and weaker than the given topology and that every point of $X$ has a neighbourhood basis in $X$ consisting of sets that are closed in $(X, \mathcal{W})$. We say that $\mathcal{W}$ is a witness topology on $X$. A Hausdorff space is called strongly zero-dimensional if the large inductive dimension is at most 0 .

REMARK 4. The spaces $\mathfrak{E}_{\mathrm{c}}(\lambda, \kappa)$ and $\left(\mathfrak{E}_{\mathrm{c}}(\lambda, \kappa)\right)^{\omega}$ are examples of almost zero-dimensional spaces. The standard witness topology on $\mathfrak{E}_{\mathrm{c}}(\lambda, \kappa)$ is the topology that is inherited from the product space $\mathbb{R}^{\kappa}$. This follows immediately from the well-known fact that sets of the form $\left\{x \in \ell_{\kappa}^{p}:\|x\| \leq r\right\}$ are closed subsets of $\mathbb{R}^{\kappa}$ and from the zero-dimensionality of the $F_{\alpha}$. Since the $F_{\alpha}$ are compact we also find that the standard witness topology on $\mathfrak{E}_{\mathrm{c}}(\lambda, \kappa)$ is $\sigma$-compact.

Definition 5. A space is called cohesive if every point has a neighbourhood that does not contain nonempty clopen subsets of the space.

REMARK 6. Erdös [10] proved that $\mathfrak{E}_{\mathrm{c}}=\mathfrak{E}_{\mathrm{c}}(\omega, \omega)$ and $\mathfrak{E}$ are not zerodimensional by showing that the spaces are cohesive. Note that the product of a cohesive space with an arbitrary space is trivially also cohesive. Both $\mathfrak{E}$ and $\mathfrak{E}_{\mathrm{c}}$ are universal spaces for the class of separable metrizable almost zero-dimensional spaces (see [5, Theorem 4.15]).

Definition 7. An arc is a space that is homeomorphic to the interval $[0,1]$. An $\mathbb{R}$-tree $\mathbb{T}$ is a metric space that is arcwise connected such that every arc is isometric to an interval in $\mathbb{R}$.

Mayer and Oversteegen [14 proved that topologically the $\mathbb{R}$-trees are precisely the metric spaces that are uniquely arcwise connected and locally arcwise connected.

Definition 8. Let $X$ be a uniquely arcwise connected metric space. If $x, y \in X$ with $x \neq y$ then $[x, y]$ denotes the unique arc in $X$ that has $x$ and $y$ as endpoints; $[x, x]$ denotes the singleton $\{x\}$. We will also use $(x, y)=[x, y] \backslash\{x, y\}$. We define the set of interior points of $X$ by $\mathfrak{i} X=$ $\bigcup\{(x, y): x, y \in X\}$. The set of all endpoints of $X$ is $\mathfrak{e} X=X \backslash \mathfrak{i} X$.

The valency of a point $x \in X$ is the cardinality of the set of components of $X \backslash\{x\}$. An $\mathbb{R}$-tree is $\kappa$-universal if any $\mathbb{R}$-tree with valency at most $\kappa$ at every point can be isometrically imbedded into it.

According to Mayer, Nikiel, and Oversteegen [13, the complete $\kappa$-universal $\mathbb{R}$-tree with valency $\kappa$ at every point is unique.

Definition 9. A function $\varphi: X \rightarrow \hat{\mathbb{R}}=[-\infty, \infty]$ is called lower semicontinuous $(L S C)$ if $\{x \in X: \varphi(x)>t\}$ is open in $X$ for every $t \in \mathbb{R}$. 
We let $\mathbb{T}_{\mathrm{w}}$ denote the $\mathbb{R}$-tree $\mathbb{T}$ equipped with the weak topology, that is, the topology generated by the subbasis

$$
\mathcal{S}=\{C: C \text { is a component of } \mathbb{T} \backslash\{x\} \text { for some } x \in \mathbb{T}\} .
$$

Note that this topology is not necessarily metric. However, analogously to the proof of [5, Lemma 3.1] it can be shown that it is a regular topology and that $\mathfrak{e} \mathbb{T}_{\mathrm{w}}$, that is, the endpoint set $\mathfrak{e} \mathbb{T}$ seen as a subspace of $\mathbb{T}_{\mathrm{w}}$, is zerodimensional. The proof of the following lemma is then completely analogous to that of [5, Lemma 3.2].

Lemma 10. Let $(\mathbb{T}, \rho)$ be an $\mathbb{R}$-tree with a convex metric. Let $p \in \mathbb{T}$ be a fixed point and let $\varphi: \mathbb{T}_{\mathrm{w}} \rightarrow \mathbb{R}^{+}$be defined by $\varphi(x)=\rho(p, x)$. Then $\varphi$ is an LSC function such that the natural projection $\pi$ from the graph of $\varphi$ to $\mathbb{T}$ is a homeomorphism. Consequently, $\mathfrak{e} \mathbb{T}_{\mathrm{w}}$ witnesses the almost zero-dimensionality of $\mathfrak{e} \mathbb{T}$.

REMARK 11 . We can now immediately draw the conclusion that ind $\mathfrak{e} \mathbb{T}$ $\leq 1$ for an arbitrary $\mathbb{R}$-tree $\mathbb{T}$. A stronger result can be found in Mayer and Oversteegen [14, Theorem 2.3], namely Ind $\mathbb{T} \leq 1$ for every $\mathbb{R}$-tree.

3. Nonseparable complete Erdős spaces are unstable. Dijkstra, van Mill, and Steprāns [6] proved that $\mathfrak{E}_{\mathrm{c}}$ is unstable, that is, $\mathfrak{E}_{\mathrm{c}}^{\omega} \not \mathfrak{E}_{\mathrm{c}}$. Their proof depends heavily on the separability of the space. Another proof can be found in Dijkstra [1, Corollary 5.8] and we are able to generalize that method to the nonseparable setting, due to our knowledge of the structure of nonseparable complete Erdős spaces (see Theorem 2 ).

Lemma 12. Let $Y$ be a separable, metrizable, almost zero-dimensional space with some witness topology $\mathcal{W}$ and define $Z=(Y, \mathcal{W})$. If $X$ is an arbitrary metrizable space, then every closed set in $Y \times X$ is a $G_{\delta}$-subset of $Z \times X$.

Proof. We will show that every open set in $Y \times X$ is an $F_{\sigma}$-set with respect to $Z \times X$. Let $O$ be an arbitrary open set in $Y \times X$. Since $Y$ is a separable, metrizable, almost zero-dimensional space, we can find a countable collection $\mathcal{B}=\left\{B_{0}, B_{1}, \ldots\right\}$ of closed subsets of $Z$ with the property that for every $x \in Y$ and for every neighbourhood $U \subset Y$ of $x$ there is some $i \in \omega$ with $x \in \operatorname{int}_{Y} B_{i} \subset B_{i} \subset U$. Since $X$ is metrizable it has a basis $\mathcal{C}=\bigcup_{i \in \omega} \mathcal{C}_{i}$ such that each $\mathcal{C}_{i}$ is locally finite. We define, for $i, j \in \omega$,

$$
\mathcal{U}_{i j}=\left\{\bar{C}: C \in \mathcal{C}_{j}, B_{i} \times \bar{C} \subset O\right\} .
$$

Note that $\mathcal{U}_{i j}$ is a locally finite collection of closed sets, thus its union $C_{i j}$ is closed in $X$. We clearly have $O=\bigcup_{i, j \in \omega} B_{i} \times C_{i j}$, thus $O$ is an $F_{\sigma}$-subset of $Z \times X$. 
A space is called $\sigma$-complete if it can be written as a countable union of topologically complete spaces.

Proposition 13. If $\mathcal{W}$ is a topology on $\mathfrak{E}_{\mathrm{c}}^{\omega}$ that witnesses the almost zero-dimensionality of $\mathfrak{E}_{\mathrm{c}}^{\omega}$, then $\emptyset$ is the only open subset of $\mathfrak{E}_{\mathrm{c}}^{\omega}$ that is $\sigma$-complete as a subspace of $\left(\mathfrak{E}_{\mathrm{c}}^{\omega}, \mathcal{W}\right)$.

Proof. Suppose that $O \subset \mathfrak{E}_{\mathrm{c}}^{\omega}$ is nonempty and open and that $O=\bigcup_{i=1}^{\infty} G_{i}$ for topologically complete subspaces $G_{i}$ of $\left(\mathfrak{E}_{\mathrm{c}}^{\omega}, \mathcal{W}\right)$. Put $G_{0}=\emptyset$. We construct by recursion a strictly increasing sequence $j_{0}, j_{1}, \ldots \in \omega$ and a sequence $x_{0}, x_{1}, \ldots \in \mathfrak{E}_{\mathrm{c}}$ such that for every $i \in \omega$ we find

$$
A_{i}=\left\{\left(x_{0}, \ldots, x_{j_{i}}\right)\right\} \times \prod_{k=j_{i}+1}^{\infty} \mathfrak{E}_{\mathrm{c}} \subset O \backslash G_{i} .
$$

Let $a \in O$; then there exists $j_{0} \geq 0$ such that

$$
\left\{\left(a_{0}, \ldots, a_{j_{0}}\right)\right\} \times \prod_{k=j_{0}+1}^{\infty} \mathfrak{E}_{\mathrm{c}} \subset O=O \backslash G_{0},
$$

so we can put $x_{i}=a_{i}$ for $i \leq j_{0}$.

Now assume that $j_{0}, \ldots, j_{i}, x_{0}, \ldots, x_{j_{i}}$, and $A_{i}$ have been found. Since $A_{i}$ is closed in $\mathfrak{E}_{\mathrm{c}}^{\omega}$, it is a $G_{\delta}$-subset of $\left(\mathfrak{E}_{\mathrm{c}}^{\omega}, \mathcal{W}\right)$ by Lemma 12 . We now see that $G_{i+1} \cap A_{i}$ is a topologically complete subspace of $\left(\mathfrak{E}_{\mathrm{c}}^{\omega}, \mathcal{W}\right)$, just as $G_{i+1}$. Furthermore, $A_{i}$ is known to be cohesive as a product of copies of $\mathfrak{E}_{\mathrm{c}}$ (see Remark 6), thus there is an open cover $\mathcal{U}$ of $A_{i}$ such that every element of $\mathcal{U}$ fails to contain nonempty clopen sets of $A_{i}$. Since $\mathcal{W}$ is a witness topology, we can refine $\mathcal{U}$ to a cover $\mathcal{V}$ consisting of $\mathcal{W}$-closed sets such that their interiors in $A_{i}$ cover $A_{i}$. Since $A_{i}$ is Lindelöf we may assume that $\mathcal{V}$ is countable. Note that the elements of $\mathcal{V}$ have empty interiors with respect to the zero-dimensional witness topology because otherwise they would contain nonempty clopen subsets of $A_{i}$. Since $\mathcal{V}$ is a countable closed cover, we conclude that $\left(A_{i}, \mathcal{W}\right)$ is a first category space. Since $G_{i+1} \cap A_{i}$ is a complete space with respect to the topology $\mathcal{W}$, it cannot be dense in $\left(A_{i}, \mathcal{W}\right)$. Thus we can find an $x^{\prime} \in A_{i}$ and an open subset $U$ of $\left(A_{i}, \mathcal{W}\right)$ such that $x^{\prime} \in U \subset$ $A_{i} \backslash G_{i+1}$. By the properties of the product topology and the fact that $U$ is also open in $A_{i}$ we can find a $j_{i+1}>j_{i}$ and $x_{j_{i}+1}, x_{j_{i}+2}, \ldots, x_{j_{i+1}} \in \mathfrak{E}_{\mathrm{c}}$ such that

$$
A_{i+1}=\left\{\left(x_{0}, \ldots, x_{j_{i+1}}\right)\right\} \times \prod_{k=j_{i+1}+1}^{\infty} \mathfrak{E}_{\mathrm{c}} \subset U \subset O \backslash G_{i+1} .
$$

This completes the recursion. Now

$$
x=\left(x_{0}, x_{1}, \ldots\right) \in \bigcap_{i=0}^{\infty} A_{i} \subset O \backslash \bigcup_{i=1}^{\infty} G_{i} .
$$

Since the $G_{i}$ cover $O$ we have a contradiction. 
Theorem 14. Let $X$ be a zero-dimensional, $\sigma$-complete, metrizable space. Then $\mathfrak{E}_{\mathrm{c}}^{\omega}$ cannot be imbedded as a closed subspace in $\mathfrak{E}_{\mathrm{c}} \times X$.

Proof. Note that the standard witness topology on $\mathfrak{E}_{\mathrm{c}}$, say $\mathcal{W}$, is $\sigma$ compact and metrizable; see Remark 4 . Hence the witness space $\left(\mathfrak{E}_{\mathrm{c}}, \mathcal{W}\right) \times X$ for $\mathfrak{E}_{\mathrm{c}} \times X$ is $\sigma$-complete. Now suppose there is some closed subspace $A \subset$ $\mathfrak{E}_{\mathrm{c}} \times X$ which is homeomorphic to $\mathfrak{E}_{\mathrm{c}}^{\omega}$. The subspace topology inherited by $A$ from $\left(\mathfrak{E}_{\mathrm{c}}, \mathcal{W}\right) \times X$ is then a witness to the almost zero-dimensionality of $A$; let us denote this topology by $\mathcal{W}^{\prime}$. The closed subspace $A$ is a $G_{\delta}$-subset of the $\sigma$-complete space $\left(\mathfrak{E}_{\mathrm{c}}, \mathcal{W}\right) \times X$, by Lemma 12 . Hence $\left(A, \mathcal{W}^{\prime}\right)$ is $\sigma$-complete as well. We now have a contradiction with Proposition 13 .

Corollary 15. If $X$ is a zero-dimensional, $\sigma$-complete, metrizable space, then $\mathfrak{E}_{\mathrm{c}} \times X \not \approx \mathfrak{E}_{\mathrm{c}}^{\omega} \times X$ and $\mathfrak{E}_{\mathrm{c}} \times X \not \approx\left(\mathfrak{E}_{\mathrm{c}} \times X\right)^{\omega}$.

Proof. The space $\mathfrak{E}_{\mathrm{c}}^{\omega} \times X$ contains a closed copy of $\mathfrak{E}_{\mathrm{c}}^{\omega}$, whereas $\mathfrak{E}_{\mathrm{c}} \times X$ fails to contain such a copy, by Theorem 14 .

Remark 16. Note that in [5, Proposition 9.1 and Corollary 9.5] Dijkstra and van Mill prove that $\mathfrak{E}_{\mathrm{c}} \times \mathbb{Q}^{\omega} \approx \mathfrak{E} \approx \mathfrak{E}_{\mathrm{c}}^{\omega} \times \mathbb{Q}^{\omega}$, from which we conclude that $\sigma$-completeness in Theorem 14 and Corollary 15 is essential.

TheOREM 17. Nonseparable complete Erdös spaces are unstable: if $\omega \leq$ $\lambda \leq \kappa$, then $\mathfrak{E}_{\mathrm{c}}(\lambda, \kappa) \not \approx\left(\mathfrak{E}_{\mathrm{c}}(\lambda, \kappa)\right)^{\omega}$.

Proof. By Theorem 2 we have $\mathfrak{E}_{\mathrm{c}}(\lambda, \kappa) \approx \mathfrak{E}_{\mathrm{c}} \times\left(\lambda_{\mathrm{D}}\right)^{\omega} \times \kappa_{\mathrm{D}}$, to which we can apply Corollary 15.

The fact that the spaces $\mathfrak{E}_{\mathrm{c}}(\lambda, \kappa)$ are unstable, just like $\mathfrak{E}_{\mathrm{c}}$, confirms us in our belief that the spaces $\mathfrak{E}_{\mathrm{c}}(\lambda, \kappa)$ as introduced and studied in [7] are correct generalizations of complete Erdös space to the nonseparable setting.

4. Endpoint sets of certain $\mathbb{R}$-trees. In [11] Kawamura, Oversteegen, and Tymchatyn sketch a proof that the endpoint set of the universal separable $\mathbb{R}$-tree as constructed in $\left[13\right.$ is homeomorphic to $\mathfrak{E}_{\mathrm{c}}$. The result is generalized by Dijkstra and van Mill in [4, Theorem 4.5]. However, it cannot be generalized to the nonseparable case, since the generalizations of the universal separable $\mathbb{R}$-tree do not have any endpoints. We will show some explicit $\mathbb{R}$-trees that can be imbedded in this $\kappa$-universal $\mathbb{R}$-tree with endpoint sets that are dense in the tree and homeomorphic to $\mathfrak{E}_{\mathrm{c}}(\kappa, \kappa)$, the nonseparable complete Erdös space of local weight and weight equal to $\kappa$. Unfortunately, some universality properties of $\mathfrak{E}_{\mathrm{c}}$ regarding endpoint sets of $\mathbb{R}$-trees cannot be generalized; see Proposition 31.

In [8], Dyubina and Polterovich present an explicit construction of the unique $\kappa$-universal $\mathbb{R}$-tree described in $\S 2$, as follows. 
Definition 18. Let $\kappa$ be an infinite cardinal number and let $\tilde{A}_{\kappa}$ be the set of all functions $f:\left[0, \rho_{f}\right) \rightarrow \kappa_{\mathrm{D}}$, where $\rho_{f} \geq 0$, that are piecewise constant from the right, that is, for each $t \in\left[0, \rho_{f}\right)$ there exist $\varepsilon>0$ such that $f\left\lceil[t, t+\varepsilon]\right.$ is constant. The distance between $f, \tilde{f} \in \tilde{A}_{\kappa}$ is denoted by

$$
d(f, \tilde{f})=\rho_{f}+\rho_{\tilde{f}}-2 s_{f, \tilde{f}},
$$

where $s_{f, \tilde{f}}$ is the separation moment between the functions $f$ and $\tilde{f}$ :

$$
s_{f, \tilde{f}}=\sup \left\{t: f\left(t^{\prime}\right)=\tilde{f}\left(t^{\prime}\right) \forall t^{\prime}<t\right\} .
$$

In particular, if $\rho_{f}=0$ we consider the function $f=\emptyset \in \tilde{A}_{\kappa}$. We also assume that $0 \in \kappa$.

REMARK 19. The weight and cardinality of $\tilde{A}_{\kappa}$ are $\kappa^{\omega}$ and in particular we have $w\left(\tilde{A}_{\kappa}\right)=\mathfrak{c}$ whenever $\kappa \leq \mathfrak{c}$. Define

$$
\begin{aligned}
A=\left\{f \in \tilde{A}_{\kappa}: \rho_{f}=2, f \uparrow[1,2) \equiv 0,\right. & \\
& \left.\forall n \in \omega, f \uparrow\left[1-2^{-n}, 1-2^{-n-1}\right) \text { is constant }\right\}
\end{aligned}
$$

and note that $|A|=\kappa^{\omega}$. If $f, g \in A$ and $f \neq g$, then $f \uparrow[0,1) \neq g \uparrow[0,1)$, thus $d(f, g)>2$. We see that $A$ is discrete and that $w\left(\tilde{A}_{\kappa}\right) \geq \kappa^{\omega}$. On the other hand, note that if $f \in \tilde{A}_{\kappa}$ then the set $D_{f}$ of points in $\left[0, \rho_{f}\right)$ where $f$ is discontinuous is a wellordered and hence countable subset of $[0, \infty)$. Since $f$ is fully characterized by its restriction to $\{0\} \cup D_{f}$ and the value of $\rho_{f}$, we have $\left|\tilde{A}_{\kappa}\right| \leq\left|([0, \infty) \times \kappa)^{\omega} \times[0, \infty)\right|=\kappa^{\omega}$. Since $\tilde{A}_{\kappa}$ is metric we find that its weight is at most $\kappa^{\omega}$.

Note that $\tilde{A}_{\kappa}$ does not have any endpoints and is nonseparable, thus $\tilde{A}_{\omega}$ is not the (unique) universal separable $\mathbb{R}$-tree. However, the universal separable $\mathbb{R}$-tree can be isometrically imbedded in $\tilde{A}_{\omega}$. It follows easily from the Uniqueness Theorem [13, Theorem 2.5] that the subspace of $\tilde{A}_{\omega}$ consisting of those functions that are only discontinuous at points in $\mathbb{Q}$, with the extra condition that for every $t \in\left(0, \rho_{f}\right)$ there exist $\delta>0$ with $f\lceil[t-\delta, t)$ constant as well, is the universal separable $\mathbb{R}$-tree.

Definition 20. Let $G \subset(0, \infty)$ be an additive semigroup of $\mathbb{R}$. Define $\mathbb{T}(G, \kappa)$ to be the subspace of $\tilde{A}_{\kappa}$ consisting of all functions $f$ that are only discontinuous at points in $G$ with the extra condition that for every $t \in\left(0, \rho_{f}\right)$ there exist $\delta>0$ such that $f\lceil[t-\delta, t)$ is constant. For convenience, we write $\mathbb{T}_{\kappa}=\mathbb{T}((0, \infty), \kappa)$.

Remark 21. The space $\mathbb{T}(G, \kappa)$ defined above is an $\mathbb{R}$-tree. Each function $f \in \mathfrak{i} \mathbb{T}(G, \kappa)$ corresponds to a strictly increasing sequence $\left(y_{i}\right)_{i=0}^{n} \subset G$ for some $n$ with $y_{n}=\rho_{f}$ and a sequence $\left(\alpha_{i}\right)_{i=0}^{n}$ such that $\alpha_{i} \neq \alpha_{i+1}$, in the sense that $f$ is of the form $f(x)=\alpha_{0}$ if $x \in\left[0, y_{0}\right)$ and $f(x)=\alpha_{i}$ if $x \in\left[y_{i-1}, y_{i}\right)$ for $1 \leq i \leq n$. 
Furthermore, each $f \in \mathfrak{e} \mathbb{T}(G, \kappa)$ corresponds to a strictly increasing sequence $\left(y_{i}\right)_{i \in \omega} \subset G$ which converges to $\rho_{f}$ and a sequence $\left(\alpha_{i}\right)_{i \in \omega}$ such that $\alpha_{i} \neq \alpha_{i+1}$, in the sense that $f$ is of the form $f(x)=\alpha_{0}$ if $x \in\left[0, y_{0}\right)$ and $f(x)=\alpha_{i}$ if $x \in\left[y_{i-1}, y_{i}\right)$ for $i \in \omega$. Hence the endpoint set of an $\mathbb{R}$-tree $\mathbb{T}(G, \kappa)$ is dense in the tree.

Note that $\mathbb{T}(\mathbb{Q} \cap(0, \infty), \omega)$ is the universal separable $\mathbb{R}$-tree and that by Kawamura, Oversteegen, and Tymchatyn [11] its endpoint set $\mathfrak{e} \mathbb{T}(\mathbb{Q} \cap(0, \infty), \omega)$ is homeomorphic to complete Erdős space $\mathfrak{E}_{\mathrm{c}}$.

We put

$$
Z_{\kappa}=\left\{\alpha=\left(\alpha_{i}\right)_{i \in \omega} \in\left(\kappa_{\mathrm{D}}\right)^{\omega}: \alpha_{i} \neq \alpha_{i+1} \text { for every } i \in \omega\right\},
$$

seen as a subspace of the strongly zero-dimensional product space $\left(\kappa_{\mathrm{D}}\right)^{\omega}$.

LEMMA $22 . Z_{\kappa} \approx\left(\kappa_{\mathrm{D}}\right)^{\omega}$.

Proof. Note that $Z_{\kappa}$ is a $G_{\delta}$-subspace of the completely metrizable space $\left(\kappa_{\mathrm{D}}\right)^{\omega}$. The space $Z_{\omega}$ is a completely metrizable separable space such that every compact subset has an empty interior. According to the Aleksandrov-Urysohn Theorem (e.g. [12, Theorem 7.7]) this is precisely the case when a space is homeomorphic to $\left(\omega_{\mathrm{D}}\right)^{\omega}$. If $\kappa>\omega$, then the space $Z_{\kappa}$ is clearly a strongly zero-dimensional completely metrizable space of weight $\kappa$ such that $w(U)=\kappa$ for all nonempty open subsets $U$. Hence by a theorem of Stone [15], it is homeomorphic to $\left(\kappa_{\mathrm{D}}\right)^{\omega}$.

Definition 23. Let $G \subset \mathbb{R}$. We define the metric space $(M(G), \rho)$ by

$$
M(G)=\left\{x=\left(x_{i}\right)_{i \in \omega} \in\left(G_{\mathrm{D}}\right)^{\omega}: \sum_{i \in \omega}\left|x_{i}\right|<\infty\right\}
$$

and

$$
\rho(x, \tilde{x})=\sum_{i \in \omega} 2^{-i} D\left(x_{i}, \tilde{x}_{i}\right)+\sum_{i \in \omega}\left|x_{i}-\tilde{x}_{i}\right|,
$$

where $x, \tilde{x} \in M(G)$ and $D$ denotes the discrete metric that only assumes the values 0 and 1 .

Observe that the topology on $M(G)$ is generated by the product topology on $\left(G_{\mathrm{D}}\right)^{\omega}$ in combination with the 1 -norm from the Banach space $\ell_{\omega}^{1}$. The space $M(\mathbb{R})$ features in $[7]$ where it is called $M$ and shown to be homeomorphic to $\mathfrak{E}_{\mathrm{c}}(\mathfrak{c}, \mathfrak{c})$. A completely analogous argument proves the following result.

Proposition 24. If $G$ is an infinite subset of $\mathbb{R}$ such that $G=2 G$ then $M(G) \approx \mathfrak{E}_{\mathrm{c}}(|G|,|G|)$.

Proposition 25. If $G \subset(0, \infty)$ is an additive semigroup of $\mathbb{R}$, then $\mathfrak{e} \mathbb{T}(G, \kappa) \approx M(G) \times Z_{\kappa}$. 
Proof. For $(x, \alpha) \in M(G) \times Z_{\kappa}$ we let $h(x, \alpha)$ be the function $f$ : $\left[0, \sum_{i \in \omega} x_{i}\right) \rightarrow \kappa_{\mathrm{D}}$ such that $f(z)=\alpha_{i}$ whenever $z \in\left[\sum_{j=0}^{i-1} x_{j}, \sum_{j=0}^{i} x_{j}\right)$. This assignment makes $h: M(G) \times Z_{\kappa} \rightarrow \mathfrak{e} \mathbb{T}(G, \kappa)$ a bijection. We first prove the continuity of $h$. Let $(x, \alpha) \in M(G) \times Z_{\kappa}$ and $\varepsilon>0$ be arbitrary. Choose an $m \in \mathbb{N}$ such that $\sum_{i \geq m} x_{i}<\varepsilon / 3$. Let $O$ be the set of all $\left(\left(\tilde{x}_{i}\right)_{i \in \omega},\left(\tilde{\alpha}_{i}\right)_{i \in \omega}\right) \in M(G) \times Z_{\kappa}$ such that $x_{i}=\tilde{x}_{i}$ and $\alpha_{i}=\tilde{\alpha}_{i}$ for $i<m$ and $\sum_{i \in \omega}\left|x_{i}-\tilde{x}_{i}\right|<\varepsilon / 3$. Then for every $(\tilde{x}, \tilde{\alpha}) \in O$ we have

$$
\begin{aligned}
d(h(x, \alpha), h(\tilde{x}, \tilde{\alpha})) & \leq \sum_{i \geq m} x_{i}+\sum_{i \geq m} \tilde{x}_{i}<\varepsilon / 3+\sum_{i \geq m}\left|\tilde{x}_{i}-x_{i}\right|+\sum_{i \geq m} x_{i} \\
& <\varepsilon / 3+\varepsilon / 3+\varepsilon / 3=\varepsilon .
\end{aligned}
$$

Now let us prove that $h$ is open. Again let $(x, \alpha) \in M(G) \times Z_{\kappa}$ be arbitrary. Consider an open neighbourhood $U$ of this point. Without loss of generality, we may assume that $U$ is the set of all $(\tilde{x}, \tilde{\alpha})$ for which the first $m$ coordinates of $\tilde{x}$ and $\tilde{\alpha}$ coincide with those of $x$ and $\alpha$ and $\sum_{i \in \omega}\left|x_{i}-\tilde{x}_{i}\right|<\varepsilon$. Let $n$ be an integer for which $\sum_{i>n} x_{i}<\varepsilon$ and $n \geq m$ and define $\delta=$ $\sum_{i \geq n} x_{i}>0$. Suppose that $d(h(x, \alpha), \tilde{f})<\delta$, where $\tilde{f}=h(\tilde{x}, \tilde{\alpha})$. For some $k \in \omega$, we then have $x_{i}=\tilde{x}_{i}$ for every $i<k-1$ and $\alpha_{i}=\tilde{\alpha}_{i}$ for every $i<k$, and

$$
d(h(x, \alpha), \tilde{f}) \leq\left|x_{k-1}-\tilde{x}_{k-1}\right|+\sum_{i \geq k} x_{i}+\sum_{i \geq k} \tilde{x}_{i}<\delta .
$$

Hence $k>n \geq m$ and $x_{i}$ and $\tilde{x}_{i}$ as well as $\alpha_{i}$ and $\tilde{\alpha}_{i}$ coincide on at least the first $m$ coordinates. Moreover,

$$
\sum_{i \in \omega}\left|x_{i}-\tilde{x}_{i}\right|=\sum_{i \geq k-1}\left|x_{i}-\tilde{x}_{i}\right| \leq\left|x_{k-1}-\tilde{x}_{k-1}\right|+\sum_{i \geq k} x_{i}+\sum_{i \geq k} \tilde{x}_{i}<\delta<\varepsilon .
$$

This implies that $\tilde{f} \in h(U)$, whence $h(U)$ is open.

ThEOREM 26. The endpoint set of the $\mathbb{R}$-tree $\mathbb{T}_{\kappa}$ is homeomorphic to $\mathfrak{E}_{\mathrm{c}}(\mathfrak{c}+\kappa, \mathfrak{c}+\kappa)$ and dense in $\mathbb{T}_{\kappa}$.

Proof. Put $G=(0, \infty)$. Using Propositions 25, 24, Lemma 22, and Theorem 2 we find

$$
\begin{aligned}
\mathfrak{e} \mathbb{T}_{\kappa} & \approx M(G) \times Z_{\kappa} \approx \mathfrak{E}_{\mathrm{c}}(\mathfrak{c}, \mathfrak{c}) \times\left(\kappa_{\mathrm{D}}\right)^{\omega} \\
& \approx \mathfrak{E}_{\mathrm{c}} \times\left(\mathfrak{c}_{\mathrm{D}}\right)^{\omega} \times\left(\kappa_{\mathrm{D}}\right)^{\omega} \approx \mathfrak{E}_{\mathrm{c}}(\mathfrak{c}+\kappa, \mathfrak{c}+\kappa) .
\end{aligned}
$$

The same argument also proves the following result.

Proposition 27. Let $\kappa \leq \mathfrak{c}$ and let $G$ be an additive semigroup in $(0, \infty)$ such that $G=2 G$ and $\kappa=|\bar{G}|$. Then the endpoint set of the $\mathbb{R}$-tree $\mathbb{T}(G, \kappa)$ is homeomorphic to $\mathfrak{E}_{\mathrm{c}}(\kappa, \kappa)$.

In particular, if we choose $G=\mathbb{Q} \cap(0, \infty)$ then $\mathbb{T}(G, \omega)$ is the universal separable $\mathbb{R}$-tree and we have another proof of the known fact (due to 
Kawamura, Oversteegen, and Tymchatyn [11]) that the endpoint set of the universal separable $\mathbb{R}$-tree is homeomorphic to $\mathfrak{E}_{\mathrm{c}}$.

Let $\mathbb{T}$ be an arbitrary $\mathbb{R}$-tree, which we may assume to be a subtree of some $\kappa$-universal $\mathbb{R}$-tree $\tilde{A}_{\kappa}$. Consider an extension $\mathbb{T}^{\prime}$ of $\mathbb{T}$ that is obtained by adding for every point $x \in \mathbb{T} \backslash \mathbb{T}$ an isometric copy of $[0, \infty)$ that lies in $\tilde{A}_{\kappa} \backslash \mathbb{T}$ and whose endpoint is $x$. It is easily verified that $\mathbb{T}^{\prime}$ is complete and that $\mathfrak{e T}^{\prime}=\mathfrak{e} \mathbb{T}$. Thus every endpoint set of an $\mathbb{R}$-tree is an endpoint set of some complete $\mathbb{R}$-tree. It is shown in [7] that every space of the form $\mathcal{E}_{\mu}$ as described in the introduction can be imbedded in $\mathfrak{E}_{\mathrm{c}}(\mu, \mu)$, so with Theorem 26] we have:

Proposition 28. Every space of the form $\mathcal{E}_{\mu}$ is homeomorphic to the endpoint set of some complete $\mathbb{R}$-tree.

Consider the results stated below, which follow from [4, Proposition 4.7] respectively [7, Corollary 34].

Proposition 29. If an $\mathbb{R}$-tree $\mathbb{T}$ is separable and complete, then its endpoint set can be imbedded in $\mathfrak{E}_{\mathrm{c}}$ as a closed subspace.

Proposition 30. A space of the form $\mathcal{E}_{\mu}$ can be imbedded in $\mathfrak{E}_{\mathrm{c}}(\mu, \mu)$ as a closed subspace if and only if it is topologically complete.

We would like to look for a possible generalization of Proposition 29 to nonseparable $\mathbb{R}$-trees and the spaces $\mathfrak{E}_{\mathrm{c}}(\kappa, \kappa)$. The endpoint set of a separable complete $\mathbb{R}$-tree is topologically complete because the interior is a countable union of arcs. By the above observations, it is clear that this is not the case for nonseparable $\mathbb{R}$-trees, so at a minimum we need to add the requirement that the endpoint set is topologically complete. Consider the space $\mathfrak{E}_{\mathrm{c}}^{\omega}$ and note that it can be imbedded in $\mathfrak{E}_{\mathrm{c}}$; see Remark 6 . Since $\mathfrak{E}_{\mathrm{c}}$ can be represented as an endpoint set of a separable $\mathbb{R}$-tree, so can $\mathfrak{E}_{\mathrm{c}}^{\omega}$ (in fact, it can be arranged that every point has valency at most 3 ). With Theorem 14 we find the following result.

Proposition 31. There exists a complete $\mathbb{R}$-tree whose points have valency at most 3 with a topologically complete endpoint set that does not admit a closed imbedding in any $\mathfrak{E}_{\mathrm{c}}(\kappa, \kappa)$.

Since the trees $\mathbb{T}_{\kappa}$ have endpoints, they are not homeomorphic to the unique $\mathbb{R}$-trees that are complete and have valency $\kappa$ at every point, that is, to $\tilde{A}_{\kappa}$. Yet, it might be possible that $\mathbb{T}_{\kappa}$ still contains a copy of every $\mathbb{R}$-tree with valency at most $\kappa$ at every point. However, this is not to be expected, because by Remark 19 we have $w\left(\tilde{A}_{\kappa}\right)=\kappa^{\omega}$ and by Theorems 1 and 26 we have $w\left(\mathbb{T}_{\kappa}\right) \leq \mathfrak{c}+\kappa$. The proposition below shows that for any $\kappa$ the tree $\mathbb{T}_{\kappa}$ is not universal for $\mathbb{R}$-trees with valency at most $\kappa$ at every point. 
Proposition 32. There exists a complete $\mathbb{R}$-tree of weight $\mathfrak{c}$, and valency at most 3 at every point, that cannot be imbedded in any $\mathbb{R}$-tree $\mathbb{T}_{\kappa}$. In particular, the $\mathbb{R}$-tree $\mathbb{T}_{\kappa}$ is not universal for $\mathbb{R}$-trees with valency at most $\kappa$ at every point.

Proof. The $\mathbb{R}$-tree that cannot be imbedded in $\mathbb{T}_{\kappa}$ looks like a so-called Cantor or Gehman dendrite, with a half-line attached to every endpoint. For convenience, we consider it as a subspace of $\tilde{A}_{\omega}$, as follows. Let $0=$ $s_{0}<s_{1}<s_{2}<\cdots<1$ be such that $\lim _{n \rightarrow \infty} s_{n}=1$. We define

$$
\begin{aligned}
\mathbb{D}=\left\{f \in \tilde{A}_{\omega}: f \uparrow\left[1, \rho_{f}\right) \equiv\right. & 0 \text { and } \\
& \left.\quad f \uparrow\left[s_{n}, \min \left\{\rho_{f}, s_{n+1}\right\}\right) \equiv 0 \text { or } 1 \text { for } n \in \omega\right\} .
\end{aligned}
$$

It is clear that $\mathbb{D}$ is complete and has weight $\mathfrak{c}$. Assume the existence of an imbedding $H: \mathbb{D} \rightarrow \mathbb{T}_{\kappa}$ for a given $\kappa$. Recursively, we are going to construct functions $f_{0}, f_{1}, \ldots \in \mathbb{D}$ with $\rho_{f_{n}}=s_{n+1}$ such that for $n \in \mathbb{N}$ :

1. $f_{n} \uparrow\left[0, s_{n}\right)=f_{n-1}$

2. $H\left(f_{n}\right)$ is discontinuous at $t_{n}=\rho_{H\left(f_{n-1}\right)}$.

Consider the root $\emptyset$ of $\mathbb{D}$. The set $\mathbb{D} \backslash\{\emptyset\}$ has two components at most one of which can have the property that some elements $h$ of its image under $H$ have the property $\rho_{h}<\rho_{H(\emptyset)}$. Let $C$ be a component such that $\rho_{H(h)}>\rho_{H(\emptyset)}$ for every $h \in C$ and let $f_{0}$ be the (unique) element of $C$ such that $\rho_{f_{0}}=s_{1}$. Observe that $H\left\lceil C\right.$ is monotone in the sense that for $h, h^{\prime} \in C$ we have $h \subset h^{\prime}$ if and only if $H(h) \subset H\left(h^{\prime}\right)$.

Now suppose that $f_{n-1}$ has been found. Consider for $i=0,1$ the functions $g_{i}$, defined by $g_{i} \uparrow\left[0, s_{n}\right)=f_{n-1}, g_{i} \uparrow\left\lceil s_{n}, s_{n+1}\right) \equiv i$ and $\rho_{g_{i}}=s_{n+1}$. If $H\left(g_{0}\right)\left(t_{n}\right)=H\left(g_{1}\right)\left(t_{n}\right)$, then there exists a $\delta>0$ with $H\left(g_{0}\right)\left\lceil\left[0, t_{n}+\delta\right)=\right.$ $H\left(g_{1}\right) \uparrow\left[0, t_{n}+\delta\right)$. Then for $i=0,1$ there is an $r_{i} \in\left(s_{n}, s_{n+1}\right)$ such that $H\left(g_{0} \uparrow\left[0, r_{i}\right)\right)=H\left(g_{i}\right) \uparrow\left[0, t_{n}+\delta\right)$. Since $g_{0} \uparrow\left[0, r_{0}\right) \neq g_{1} \uparrow\left[0, r_{1}\right)$, we have a contradiction with the injectivity of $H$. Thus we have $H\left(g_{0}\right)\left(t_{n}\right) \neq H\left(g_{1}\right)\left(t_{n}\right)$ and we may choose $i$ such that $H\left(g_{i}\right)$ is discontinuous at $t_{n}$. Putting $f_{n}=g_{i}$ we find that the induction hypotheses are satisfied.

We can now define $f=\bigcup_{n \in \omega} f_{n} \in \mathbb{D}$. Then $H(f)$ is discontinuous at infinitely many points, thus it must be an endpoint of $\mathbb{T}_{\kappa}$ by Remark 21. This contradicts our assumption that $H$ is an imbedding, because $f$ is not an endpoint in $\mathbb{D}$.

As noted in Remark 11, the large inductive dimension of the endpoint set of any $\mathbb{R}$-tree never exceeds one. In the separable case, the following result from [4, Theorem 4.5, Remark 4.6] provides a sufficient condition for an $\mathbb{R}$-tree to have a one-dimensional endpoint set.

THEOREM 33. Let $(\mathbb{T}, \rho)$ be a nonempty separable $\mathbb{R}$-tree such that for each $x \in \mathbb{T}$ every component of $\mathbb{T} \backslash\{x\}$ is unbounded and every $x \in \mathfrak{e} \mathbb{T}$ has 
a neighbourhood in $\mathbb{T}$ that is complete. If $\mathfrak{e} \mathbb{T}$ is dense in $\mathbb{T}$, then $\mathfrak{e} \mathbb{T} \approx \mathfrak{E}_{\mathrm{c}}$, thus ind $\mathfrak{e} \mathbb{T}>0$.

We present an example of an $\mathbb{R}$-tree $\mathbb{T}$ that is a closed subset of $\tilde{A}_{\omega}$ for which all premises of the above theorem hold, except for separability, and which has a strongly zero-dimensional endpoint set.

Proposition 34. There exists a complete $\mathbb{R}$-tree $\mathbb{T} \subset \tilde{A}_{\omega}$ such that for every $x \in \mathbb{T}$ every component of $\mathbb{T} \backslash\{x\}$ is unbounded, $\mathfrak{e} \mathbb{T}$ is dense in $\mathbb{T}$, and Ind $\mathfrak{e} \mathbb{T}=0$.

Proof. Since the weight of the metric space $\tilde{A}_{\omega}$ is $\mathfrak{c}$ (see Remark 19), there is a $\sigma$-discrete basis $\mathcal{B}=\left\{B_{\alpha}: \alpha<\mathfrak{c}\right\}$ for $\tilde{A}_{\omega}$. We arrange that no $B_{\alpha}$ is empty. Using transfinite recursion we find functions $f_{\alpha} \in \tilde{A}_{\omega}$ for $\alpha<\mathfrak{c}$ for which the following conditions hold:

(i) $f_{\alpha} \in B_{\alpha}$;

(ii) there are $t \in\left(0, \rho_{f_{\alpha}}\right)$ and $k \in \omega$ such that $f_{\alpha}\left\lceil\left[t, \rho_{f_{\alpha}}\right) \equiv 2 k\right.$;

(iii) $f_{\alpha} \not \subset f_{\beta}$ for every $\beta<\alpha$;

(iv) if there exists an $f \in B_{\alpha}$ such that $f_{\beta} \not \subset f$ for every $\beta<\alpha$, then $f_{\beta} \not \subset f_{\alpha}$ for every $\beta<\alpha$.

Since $B_{0}$ is open, we can find an $f_{0} \in B_{0}$ for which (ii) holds. Conditions (iii) and (iv) are void in this case. For the recursion step, let $\alpha>0$ and suppose that $f_{\beta}$ is found for every $\beta<\alpha$.

CASE 1. Suppose there exists an $f \in B_{\alpha}$ such that $f_{\beta} \not \subset f$ for every $\beta<\alpha$. If it happens that $f=\emptyset$ then we replace $f$ by $f_{0} \uparrow[0, s)$ for some $s \in\left(0, \rho_{f_{0}}\right)$ that is small enough to keep the function in $B_{\alpha}$. By hypothesis (iii) we know that the new $f$ still has the property $f_{\beta} \not \subset f$ for every $\beta<\alpha$. If necessary, shorten the domain of $f$ slightly so that there is a $\delta \in\left(0, \rho_{f}\right)$ with $f\left\lceil\left[\rho_{f}-\delta, \rho_{f}\right) \equiv n\right.$ for some constant $n \in \omega$. We may assume that $\delta$ is such that $\left\{g \in \tilde{A}_{\omega}: d(f, g)<2 \delta\right\} \subset B_{\alpha}$. Since $|\alpha|<\mathfrak{c}$ and every $g \in \tilde{A}_{\omega}$ has only countably many discontinuities there exists a $t \in\left(\rho_{f}-\delta, \rho_{f}\right)$ such that no $f_{\beta}$ for $\beta<\alpha$ is discontinuous at $t$. Let $k \in \omega$ be such that $2 k \neq n$ and let $f_{\alpha} \in B_{\alpha}$ be given by $\rho_{f_{\alpha}}=t+\delta, f \uparrow[0, t)=f_{\alpha} \uparrow[0, t)$, and $\left.f_{\alpha}\right\rceil[t, t+\delta) \equiv 2 k$. So hypotheses (i) and (ii) are satisfied. Since $f_{\alpha}$ is discontinuous at $t$, no $f_{\beta}$ for $\beta<\alpha$ can extend $f_{\alpha}$ and (iii) is satisfied. If $f_{\beta} \subset f_{\alpha}$ for some $\beta<\alpha$ then for the same reason $f_{\beta} \subset f_{\alpha} \uparrow[0, t) \subset f$, which contradicts the choice of $f$. We conclude that hypothesis (iv) is also satisfied.

CASE 2. Suppose that we are not in Case 1 , that is, for every $f \in B_{\alpha}$ there is an $f_{\beta}$ with $\beta<\alpha$ and $f_{\beta} \subset f$. Then by following exactly the same procedure as in the first case, but starting with an arbitrary $f \in B_{\alpha}$, we end up with an $f_{\alpha}$ that satisfies (i)-(iii). Condition (iv) is of course void in this case. 
We now define

$$
\mathbb{T}=\tilde{A}_{\omega} \backslash \bigcup_{\alpha<\mathfrak{c}}\left\{f \in \tilde{A}_{\omega}: f_{\alpha} \varsubsetneqq f\right\} .
$$

Note that $\emptyset \in \mathbb{T}$. Since every set of the form $\left\{f \in \tilde{A}_{\omega}: f_{\alpha} \varsubsetneqq f\right\}$ is open in $\tilde{A}_{\omega}$, the set $\mathbb{T}$ is closed in $\tilde{A}_{\omega}$. Note that whenever $g \in \mathbb{T}$, also $f \in \mathbb{T}$ for every $f \subset g$, which means that $[\emptyset, g] \subset \mathbb{T}$. Thus $\mathbb{T}$ is a closed subtree of $\tilde{A}_{\omega}$ and a complete $\mathbb{R}$-tree.

We first show that $\mathfrak{e} \mathbb{T}=\left\{f_{\alpha}: B_{\alpha} \in \mathcal{B}\right\} \cap \mathbb{T}$. For every $\alpha<\mathfrak{c}$ we have $\left\{f: f_{\alpha} \varsubsetneqq f\right\} \cap \mathbb{T}=\emptyset$, hence $f_{\alpha} \notin \mathfrak{i} \mathbb{T}$ and $\left\{f_{\alpha}: B_{\alpha} \in \mathcal{B}\right\} \cap \mathbb{T} \subset \mathfrak{e} \mathbb{T}$. If $f \notin\left\{f_{\alpha}: B_{\alpha} \in \mathcal{B}\right\}, f \in \mathbb{T}$, and $k \in \omega$ is odd, then for every $t \geq 0$ we find that the function $g_{t}$ defined by $\rho_{g_{t}}=\rho_{f}+t, f \subset g_{t}$, and $g_{t} \uparrow\left[\rho_{f}, \rho_{f}+t\right) \equiv k$ is in the tree. For if there are $t, \alpha$ with $f_{\alpha} \varsubsetneqq g_{t}$, then by (ii) we have $f_{\alpha} \subset f$, which contradicts the choice of $f$. Note that $d\left(g_{s}, g_{t}\right)=|s-t|$ for all $s, t \geq 0$. Thus we have infinitely many isometric copies of $[0, \infty)$ emanating from $f$ and we conclude that $f \in \mathfrak{i} \mathbb{T}$.

To show that $\mathfrak{e} \mathbb{T}$ is dense in $\mathbb{T}$, we show that if $B_{\alpha} \cap \mathbb{T} \neq \emptyset$, then $B_{\alpha} \cap \mathfrak{e} \mathbb{T}$ $\neq \emptyset$. Indeed, by the result in the preceding paragraph we are done if $f_{\alpha} \in \mathbb{T}$. First note that by (iv) and the definition of $\mathbb{T}, B_{\alpha} \cap \mathbb{T} \neq \emptyset$ implies that $f_{\beta} \not \subset f_{\alpha}$ for each $\beta<\alpha$. Hypothesis (iii) gives that $f_{\beta} \not \subset f_{\alpha}$ for every $\beta>\alpha$. We conclude that $f_{\alpha} \in \mathbb{T}$.

Since $\mathcal{B}$ is a $\sigma$-discrete collection in $\tilde{A}_{\omega}$, we can write $\mathcal{B}=\bigcup_{n \in \omega} \mathcal{B}_{n}$, where every collection $\mathcal{B}_{n}$ is discrete. We then find that $\left\{f_{\alpha}: B_{\alpha} \in \mathcal{B}_{n}\right\} \cap \mathbb{T}$ is a discrete and closed subspace of $\mathbb{T}$ for every $n$. Hence by [9, Theorem 2.2.7], $\mathfrak{e} \mathbb{T}=\left\{f_{\alpha}: B_{\alpha} \in \mathcal{B}\right\} \cap \mathbb{T}$ is a strongly zero-dimensional subspace of $\mathbb{T}$.

Finally, let $f \in \mathbb{T}$ be arbitrary and suppose that $C$ is a component of $\mathbb{T} \backslash\{f\}$. Since $C$ is open in $\mathbb{T}$, we can select a $g \in C \cap \mathfrak{i} \mathbb{T}$. It is shown above that infinitely many isometric copies of $[0, \infty)$ emanate from $g$ in $\mathbb{T}$, and at most one of them can contain $f$. Thus all the other infinite rays are contained in $C$ and $C$ is unbounded.

Acknowledgments. The research of the second author was supported by the Netherlands Organisation for Scientific Research (NWO), under grant 613.000 .551$.

\section{References}

[1] J. J. Dijkstra, Characterizing stable complete Erdős space, Israel J. Math., to appear.

[2] J. J. Dijkstra and J. van Mill, Homeomorphism groups of manifolds and Erdös space, Electron. Res. Announc. Amer. Math. Soc. 10 (2004), 29-38.

[3] - - - A counterexample concerning line-free groups and complete Erdös space, Proc. Amer. Math. Soc. 134 (2006), 2281-2283. 
[4] J. J. Dijkstra and J. van Mill, Characterizing complete Erdös space, Canad. J. Math. 61 (2009), 124-140.

[5] - - - Erdös space and homeomorphism groups of manifolds, Mem. Amer. Math. Soc., in press.

[6] J. J. Dijkstra, J. van Mill, and J. Steprāns, Complete Erdős space is unstable, Math. Proc. Cambridge Philos. Soc. 137 (2004), 465-473.

[7] J. J. Dijkstra, J. van Mill, and K. I. S. Valkenburg, On nonseparable Erdös spaces, J. Math. Soc. Japan 60 (2008), 793-818.

[8] A. Dyubina and I. Polterovich, Explicit constructions of universal $\mathbb{R}$-trees and asymptotic geometry of hyperbolic spaces, Bull. London Math. Soc. 33 (2001), 727-734.

[9] R. Engelking, Dimension Theory, PWN, Warszawa, and North-Holland, Amsterdam, 1978.

[10] P. Erdös, The dimension of the rational points in Hilbert space, Ann. of Math. 41 (1940), 734-736.

[11] K. Kawamura, L. G. Oversteegen, and E. D. Tymchatyn, On homogeneous totally disconnected 1-dimensional spaces, Fund. Math. 150 (1996), 97-112.

[12] A. S. Kechris, Classical Descriptive Set Theory, Grad. Texts in Math. 156, Springer, New York, 1995.

[13] J. C. Mayer, J. Nikiel, and L. G. Oversteegen, Universal spaces for R-trees, Trans. Amer. Math. Soc. 334 (1992), 411-432.

[14] J. C. Mayer and L. G. Oversteegen, A topological characterization of R-trees, ibid. 320 (1990), 395-415.

[15] A. H. Stone, Non-separable Borel sets, Rozprawy Mat. 28 (1962).

Jan J. Dijkstra, Kirsten I. S. Valkenburg

Faculteit der Exacte Wetenschappen/Afdeling Wiskunde

Vrije Universiteit

De Boelelaan 1081a

1081 HV Amsterdam, The Netherlands

E-mail: dijkstra@cs.vu.nl

kivalken@cs.vu.nl

Received 21 May 2008;

in revised form 11 November 2009 